A Review of Network and Computer Analysis of Epileptiform Discharge Free EEG to Characterize and Detect

\title{
Epilepsy
}

West, C

http://hdl.handle.net/10026.1/17118

10.1177/15500594211008285

Clinical EEG and Neuroscience

SAGE Publications

All content in PEARL is protected by copyright law. Author manuscripts are made available in accordance with publisher policies. Please cite only the published version using the details provided on the item record or document. In the absence of an open licence (e.g. Creative Commons), permissions for further reuse of content should be sought from the publisher or author. 



\section{A Review of Network and Computer Analysis of Epileptiform Discharge Free EEG to Characterize and Detect Epilepsy}

Clinical EEG and Neuroscience

$1-4$

(C) EEG and Clinical Neuroscience

Society (ECNS) 2021

Article reuse guidelines:

sagepub.com/journals-permissions

DOI: $10.1177 / 15500594211008285$

journals.sagepub.com/home/eeg

(9SAGE

\author{
(aitlin West', Wessel Woldman ${ }^{2}$, Katy Oak ${ }^{3}$, Brendan McLean $^{3}$ (D), \\ GQI and Rohit Shankar ${ }^{1,4}$ iD
}

\begin{abstract}
G tives. There is emerging evidence that network/computer analysis of epileptiform discharge free electroencephalograms AQI (EEGs) can be used to detect epilepsy, improve diagnosis and resource use. Such methods are automated and can be performed GQ4 on shorter recordings of EEG. We assess the evidence and its strength in the area of seizure detection from network/computer GQ5 analysis of epileptiform discharge free EEG. Methods. A scoping review using Preferred Reporting Items for Systematic Reviews and Meta-Analyses (PRISMA) guidance was conducted with a literature search of Embase, Medline and PsychINFO. Predesigned inclusion/exclusion criteria were applied to selected articles. Results. The initial search found 3398 articles. After duplicate removal and screening, 59I abstracts were reviewed, 64 articles were selected and read leading to 20 articles meeting the requisite inclusion/exclusion criteria. These were 9 reports and 2 cross-sectional studies using network analysis to compare and/or classify EEG. One review of 17 reports and 10 cross-sectional studies only aimed to classify the EEGs. One cross-sectional study discussed EEG abnormalities associated with autism. Conclusions. Epileptiform discharge free EEG features derived from network/ computer analysis differ significantly between people with and without epilepsy. Diagnostic algorithms report high accuracies and could be clinically useful. There is a lack of such research within the intellectual disability (ID) and/or autism populations, where epilepsy is more prevalent and there are additional diagnostic challenges.
\end{abstract}

\title{
Keywords
}

network analysis, computer analysis, diagnostic algorithms, artificial intelligence

Received December 7, 2019; revised August 18, 2020; accepted March 7, 2021.

\section{Introduction}

\section{Epilepsy and Electroencephalography}

Electroencephalography (EEG) records brain activity and is used to support the diagnosis of epilepsy, classify epileptic syndromes, help guide pharmacological management and in presurgical evaluation. ${ }^{1}$ The National Institute for Health and Care Excellence (NICE) recommends that a standard EEG recording is performed after 1 or 2 seizures if the clinical history suggests that the events could have an epileptic basis. $^{2}$ A standard EEG recording usually takes $20-40$ min. The use of electroencephalograms (EEGs) in epilepsy was reviewed by Smith. ${ }^{1}$ The likelihood of capturing a seizure on a standard EEG is low, and enhancing stimulus such as hyperventilation and photostimulation may be used to trigger epileptiform activity. ${ }^{1}$ Recordings are reviewed for the presence of epileptiform activity; most commonly they contain interictal epileptiform discharges (IEDs), which are present on the first EEG recording in up to $65 \%$ of people subsequently diagnosed with epilepsy. Repeat, sleep-deprived and prolonged EEG recordings increase sensitivity to $80 \%$ to $90 \% .{ }^{1}$ An EEG has a relatively high specificity (78\%-98\%) but low sensitivity (25\%-56\%) for the diagnosis of epilepsy. ${ }^{1}$

\section{Network and Computer EEG Analysis}

Automated computerized methods, which include network analysis and machine learning, are being used to classify EEG from shorter recordings and do not require the presence of visual epileptiform discharges. ${ }^{3}$ There are machine-learning approaches, which aim to automatically identify and classify spikes, discharges, or other IEDs from long-term EEG. ${ }^{4-6}$

\footnotetext{
'Exeter Medical School, Knowledge Spa, Truro, UK

${ }^{2}$ Centre for Systems Modelling and Quantitative Biomedicine, University of Birmingham, Birmingham, UK

${ }^{3}$ Royal Cornwall Hospitals Trust Truro, Truro, UK

${ }^{4}$ Cornwall Partnership NHS Foundation Trust, Truro, UK
}

Corresponding Author:

Rohit Shankar, Chygovenck, Threemilestone Industrial Estate, Truro Cornwall TR4 9LD, UK.

Email: rohit.shankar@nhs.net 


\section{Aim}

The main aim of this study was to assess the evidence and its strength in the area of epilepsy detection from network analysis, and other computer analysis methods, of interictal EEG.

\section{Methods}

This was a scoping review conducted using PRISMA guidance. A search of Embase, Medline and PsychInfo was performed using Healthcare Databases Advanced Search with terms relating to computer/network analysis, EEG, and epilepsy. The full list of terms used is provided in Supplemental Appendix A. Results were limited to full reports published in English from January 2008 to December 2018 so only recent material was included. No other sources were used.

Screening was separately performed by 2 coauthors. After the initial screening of titles for relevant articles, abstracts were screened using predefined eligibility criteria by both and differences in selection $(n=1)$ was reconciled by consensus.

\section{Criteria}

Studies were included if they used resting-state/interictal EEG and excluded if they studied exclusively preictal/postictal and/or ictal EEG. Studies were excluded if they used functional magnetic resonance imaging (fMRI) or an investigation other than EEG for analysis and if they used the presence of IEDs to classify the EEG. Papers were included if they reviewed or included primary data.

\section{Results}

The initial search identified 3398 articles which when examined by title for relevance, and duplication resulted in 591 abstracts being screened. Sixty-four papers were read in full and the eligibility criteria applied. A flowchart of the search process is provided in Supplemental Appendix B.

Twenty articles have been included and are detailed in Supplemental Appendix C. ${ }^{7-26}$ Thematic analysis was used to analyze these papers looking into the network analysis, classification, and influence on special populations. Nine reports and 2 cross-sectional studies use network analysis to compare and/or classify EEG. The results from these papers are compared by network feature in Supplemental Appendix D. One review of 17 controlled trials and a further 10 cross-sectional reports, including 2 which use network analysis, aim to classify the EEG. One cross-sectional study also discusses EEG abnormalities associated with a special population of autism.

A review by Acharya et $\mathrm{al}^{7}$ includes promising results from 17 articles and is particularly significant to this review. Across the studies using network analysis, the differences between analysis methods, and the differences in results, particularly between papers analyzing the same network feature, are notable findings. Of these studies, a systematic review and meta-analysis by van Diessen et al, ${ }^{25}$ which includes results from 13 articles, provides particularly strong evidence regarding 2 of the network features assessed. However, these results are limited by differences in methodology across the included studies.

\section{Summary of Review Findings}

\section{Network Analysis}

In network analysis, the electrodes of the EEG are modeled as nodes of a network. ${ }^{27}$ A connection between 2 nodes is typically quantified by comparing the relationship between the signals from 2 electrodes. After a network is derived from the electrophysiological recording, graph measures can be used to capture properties of the network structures, which can then, in turn, be used as potential markers in comparing patients and controls. ${ }^{28}$ Connectivity, mean degree, path length, and clustering coefficient are the network features used most commonly across the included studies. Nine individual studies found significant differences between the interictal EEGs of people with epilepsy in the features included in Supplemental Appendix D.

A study found no significant differences in any network feature between patients and controls, but created a successful classifier through a combination of different network features. ${ }^{23}$ A later report by the same group also found no significant differences in path length and clustering coefficient, but a different network measure (minimum spanning tree) was significantly different between patients and controls. ${ }^{24}$

The results for individual network features are commonly presented as a pattern of increased/decreased feature values, dependent on anatomical location and frequency of the EEG, rather than as an overall value. An example of this is connectivity, the feature that is most commonly investigated and this was the case in 7 of the included studies. As shown in Supplemental Table 2, results vary widely. Connectivity is calculated and represented in a variety of ways, which include synchronization, coherence, and outflow. One study investigated the EEGs of people with temporal lobe epilepsy (TLE) and found that they had decreased functional connectivity in the default-mode network regions, which are reported to be involved in TLE. ${ }^{14}$ Whereas 1 study reported increased synchronization likelihood in people with epilepsy. ${ }^{11}$

Generally, individual papers report significant differences in the network features of patients and controls, but results across the 11 reports are varied. This may well be due to significant methodological heterogeneity, discussed under 'General limitations.'

The included studies suggest that network analysis can estimate differences between interictal EEG from people with epilepsy and from healthy controls. There are, however, no studies that classify EEG using network analysis, and there is little consensus on the 'best' way to apply network analysis with regard to feature calculation and selection. 


\section{Classification}

Twelve reports involve the classification of EEG. This includes 1 review of 17 articles and reports of 10 cross-sectional studies. The review ${ }^{7}$ and a further 6 cross-sectional studies ${ }^{10,12,14,16,20,23}$ highlighted that interictal and healthy EEG could be classified with over $90 \%$ accuracy. This level of accuracy suggests that computer analysis of interictal EEG could be AQ2 clinically useful. The classification studies use a wide variety of methods to differentiate the healthy and interictal EEGs, wrich include nonlinear feature extraction and network analysis.

\section{Studies in Special Populations}

There is an increased prevalence of epilepsy in people with ID $(15 \%-30 \%){ }^{29}$ Prevalence is also increased in people with autism. ${ }^{30}$ There are diagnostic challenges within these populations as an EEG recording can be distressing for some people and there may be no cooperation. Nonepileptic events may be misinterpreted which may lead to misdiagnosis. ${ }^{29}$ There are also suggestions that people with ID and/or autism may have existing EEG abnormalities that are independent of epilepsy. ${ }^{31}$ Machine learning has been applied in the area of EEG analysis for the diagnosis of autism. A recent study used a machinelearning method to distinguish EEG from children with autism and those with other neuropsychiatric diagnoses, such as attention deficit hyperactivity disorder, as well as children developing typically, with an accuracy of $95 \% .{ }^{32}$

A search was performed to look for reports that compared and/or classified interictal EEG from people with epilepsy and EEG from people without epilepsy, in the ID and/or autism populations. No reports were identified.

\section{Limitations of the Studies Reviewed}

Methodological differences between studies make comparisons difficult. Epilepsy syndrome and/or seizure type of the population considered vary across the items. Visually, epileptiform activity differs between epilepsy types. ${ }^{1}$ Classification methods are likely to need significant adaptation to different epilepsy types. Differences may arise due to choice of specific network derivation method (linear, nonlinear, phase based or amplitude based), choice of epochs and epoch length, referencing method, sampling rate, and frequency bands.

This is particularly significant, as many of the classifiers use machine learning, in which a classifier is 'trained' on a data set. Such classifiers are vulnerable to sensitization to an individual data set, which would reduce the probability of successful classification when applied to new out-of-sample EEG recordings. Seventeen articles in 1 review and a further 3 cross-sectional reports use the same EEG data set from the University of Bonn to analyze and/or test their classification method. Training and/or testing on a wider EEG data set would ensure that classifiers are applicable to a wider variety of EEG data as would be seen in a clinical setting.
The method used to record the EEG also differed between studies, and was performed with varying numbers of electrodes. The University of Bonn data set was produced from intracranial EEG, which limits the applicability of the results to standard EEG.

\section{Limitations of This Study}

This scoping review had a focus on network analysis, and it is beyond the scope of this review to provide a comparison of other methods. Articles using fMRI for analysis were excluded from this study, as EEG alone is more suitable for a standard diagnostic test (due to lower cost, etc). These studies may provide further insight into measurable differences between the brains of people with epilepsy and those without.

\section{Conclusion}

There are suggestions that network analysis can be used to differentiate interictal EEG in people with epilepsy from findings in healthy controls. Classification methods using network analysis and other methods are highly accurate in trials. Generally, the results are limited by significant methodological variability, and further research using standardized methodology and wider EEG data sets is required to assess the reproducibility of results from individual studies. There is a lack of research within the ID/autism populations, where such methods would arguably be most useful, as the prevalence of epilepsy is increased within these populations and there are additional diagnostic challenges.

\section{Acknowledgments}

The authors acknowledge Professor Sander JWL, University College Hospitals, London, UK, for providing technical support in putting together this paper.

\section{Declaration of Conflicting Interests}

The authors declared the following potential conflicts of interest with respect to the research, authorship, and/or publication of this article: $\mathrm{CW}, \mathrm{OK}$ and BMcL have no conflicts of interest to report. WW has a commercial interest and is the scientific director in the company Neuronostics (https://www.neuronostics.com/) focused on the development and commercialization of a novel and potentially revolutionary seizure susceptibility assessment technology called \#BioEP ${ }^{\mathrm{TM}}$. RS is the clinical chief investigator for the NIHR adopted study for investigating the impact of \#BioEPTM. He has no commercial stake holding in the company.

\section{Funding}

The authors received no financial support for the research, authorship and/or publication of this article.

\section{ORCID iDs}

Brendan McLean (D) https://orcid.org/0000-0003-1419-8540 


\section{Supplemental Material}

Supplemental material for this article is available online.

\section{References}

Smith S. EEG in the diagnosis, classification, and management of patients with epilepsy. J Neurol Neurosurg Psychiatry. 2005;76: ii2-ii7.

2. Overview|Epilepsies: diagnosis and management|Guidance|NICE. Nice.org.uk. https://www.nice.org.uk/guidance/cg137. Published 2019. Accessed November 2019.

3. Kramer MA, Cash SS. Epilepsy as a disorder of cortical network organization. Neuroscientist. 2012;18(4):360-372.

4. Antoniades A, Spyrou L, Martin-Lopez D, et al. Detection of interictal discharges with convolutional neural networks using discrete ordered multichannel intracranial EEG. IEEE Trans Neural Syst Rehabil Eng. 2017;25(12):2285-2294.

5. Antoniades A, Spyrou L, Took CC, Sanei S. Deep learning for epileptic intracranial EEG data. 2016 IEEE 26th International Workshop on Machine Learning for Signal Processing (MLSP), 2016; 1-6.

6. Thomas J, Comoretto L, Jin J, Dauwels J, Cash SS, Westover MB. EEG classification via convolutional neural network-based interictal epileptiform event detection. 2018 40th Annual International Conference of the IEEE Engineering in Medicine and Biology Society (EMBC), Honolulu, HI, 2018; 3148-3151.

7. Acharya U, Vinitha Sree S, Swapna G, Martis R, Suri J. Automated EEG analysis of epilepsy: a review. Knowl Based Syst. 2013;45:147-165.

8. Adebimpe A, Aarabi A, Bourel-Ponchel E, Mahmoudzadeh M, Wallois F. Functional brain dysfunction in patients with benign childhood epilepsy as revealed by graph theory. PLOS ONE. 2015;10(10):e0139228.

9. Adebimpe A, Aarabi A, Bourel-Ponchel E, Mahmoudzadeh M, Wallois F. EEG resting state functional connectivity analysis in children with benign epilepsy with centrotemporal spikes. Front Neurosci. 2016;10:143.

10. Bao FS, Gao JM, Hu J, Lie DY, Zhang Y, Oommen KJ. Automated epilepsy diagnosis using interictal scalp EEG. Conference proceedings: Annual International Conference of the IEEE Engineering in Medicine and Biology Society IEEE Engineering in Medicine and Biology Society Conference, 2009;2009:6603-6607.

11. Bartolomei F, Bettus G, Stam CJ, Guye M. Interictal network properties in mesial temporal lobe epilepsy: a graph theoretical study from intracerebral recordings. Clin Neurophysiol. 2013;124(12):2345-2353.

12. Bosl WJ, Loddenkemper T, Nelson CA. Nonlinear EEG biomarker profiles for autism and absence epilepsy. Neuropsychiatr Electrophysiol. 2017;3(1).

13. Clemens B, Puskás S, Bessenyei M, et al. EEG Functional connectivity of the intrahemispheric cortico-cortical network of idiopathic generalized epilepsy. Epilepsy Res. 2011;96(1-2):11-23.

14. Coito A, Genetti M, Iannotti GR, et al. Altered directed functional connectivity in temporal lobe epilepsy in the absence of interictal spikes: a high density EEG study. Epilepsia. 2016;57(3):402-411.

15. Colominas MA, Jomaa MESH, Humeau-Heurtier A, Jrad N, Van Bogaert P. Time-varying time-frequency complexity measures for epileptic EEG data analysis. IEEE Trans Biomed Eng. 2018; 65(8):1681-1688.

16. Goksu H. EEG Based epileptiform pattern recognition inside and outside the seizure states. Biomed Signal Process Control. 2018;43:204-215.

17. Hussain L, Aziz W, Alowibdi JS, et al. Symbolic time series analysis of electroencephalographic (EEG) epileptic seizure and brain dynamics with eye-open and eye-closed subjects during resting states. J Physiol Anthropol. 2017;36(1):21.

18. Pyrzowski J, Ossowska $\Lambda$, Sieminski M, Nyka WM. Improved diagnosis of temporal lobe epilepsy by EEG interval analysis. I. Neurol. 2013;260.

19. Rotondi F, Franceschetti S, Avanzini G, Panzica F. Altered EEG resting-state effective connectivity in drug-naïve childhood absence epilepsy. Clin Neurophysiol. 2016;127(2):1130-1137.

20. Sairamya NJ, Thomas GS, Subathra MSP, Balakrishnan R. An effective approach to classify epileptic EEG signal using local neighbor gradient pattern transformation methods. Australas Phys Eng Sci Med. 2018;41(4).

21. Schmidt H, Woldman W, Goodfellow M, et al. A computational biomarker of idiopathic generalized epilepsy from resting state EEG. Epilepsia. 2016;57(10):e200.

22. V KR, Bhardwaj S, Panda R, et al. Machine learning detects EEG microstate alterations in patients living with temporal lobe epilepsy. Seizure. 2018;61:8-13.

23. van Diessen E, Otte WM, Braun KPJ, Jansen FE, Stam CJ. Improved diagnosis in children with partial epilepsy using a multivariable prediction model based on EEG network characteristics. PLoS ONE. 2013;8(4).

24. van Diessen E, Otte WM, Stam CJ, Braun KPJ, Jansen FE. Electroencephalography based functional networks in newly diagnosed childhood epilepsies. Clin Neurophysiol. 2016; 127(6):2325-2332.

25. van Diessen E, Zweiphenning WJEM, Jansen FE, Stam CJ, Braun KPJ, Otte WM. Brain network organization in focal epilepsy: a systematic review and meta-analysis. PloS ONE. 2014;9(12): e114606.

26. Verhoeven T, Coito A, Plomp G, et al. Automated diagnosis of temporal lobe epilepsy in the absence of interictal spikes. NeuroImage Clinical. 2018;17:10-15.

27. Bassett DS, Bullmore ET. Human brain networks in health and disease. Curr Opin Neurol. 2009;22(4):340-347.

28. van Straaten E, Stam C. Structure out of chaos: functional brain network analysis with EEG, MEG, and functional MRI. Eur Neuropsychopharmacol. 2013;23(1):7-18

29. Chapman M, Iddon P, Atkinson K, et al. The misdiagnosis of epilepsy in people with intellectual disabilities: a systematic review. Seizure. 2011;20(2):101-106.

30. Juneja M, Gupta S, Thakral A. Prevalence of unrecognized autism spectrum disorders in epilepsy: a clinic-based study. $J$ Pediatr Neurosci. 2018;13(3):308-312.

31. Buckley AW, Holmes GL. Epilepsy and autism. Cold Spring Harb Perspect Med. 2016;6(4):a022749.

32. Grossi E, Buscema M, Della Torre F, Swatzyna RJ. The "MS-ROM/IFAST" model, a novel parallel nonlinear EEG analysis technique, distinguishes ASD subjects from children affected with other neuropsychiatric disorders with high degree of accuracy. Clin EEG Neurosci. 2019;50(5):319-331. 